Page $\mathbf{1}$ of $\mathbf{2 4}$

\title{
Zinc Delivery from Non-woven Fibres within a Therapeutic Nipple Shield \\ Theresa Maier ${ }^{\mathrm{a}, \mathrm{b}}$, Rebekah L. Scheuerle ${ }^{\mathrm{a}}$, Daniel Markl ${ }^{\mathrm{a}}$, Sylvaine Bruggraber ${ }^{\mathrm{c}}$, Axel Zeitler ${ }^{\mathrm{a}}$, Ljiljana Fruk ${ }^{\mathrm{a}}$, Nigel K. H. Slater
}

${ }^{\text {a }}$ University of Cambridge, Department of Chemical Engineering and Biotechnology, West Cambridge Site, Philippa Fawcett Drive, Cambridge CB3 0AS, United Kingdom

b University of Cambridge, Department of Paediatrics, Cambridge Biomedical Campus, Cambridge CB2 0QQ, UK

c Medical Research Council Elsie Widdowson Laboratory, 120 Fulbourn Road, Cambridge CB1 9NL, United Kingdom

\section{Corresponding author}

Name: $\quad$ Theresa Maier

Address: Department of Chemical Engineering and Biotechnology, University of Cambridge

West Cambridge Site, Philippa Fawcett Drive, Cambridge CB3 0AS, UK

Telephone number: $\quad+44(0) 7778627858$

Fax number: $\quad$ +44(0)1223334796

E-mail address: tm520@cam.ac.uk 
Page $\mathbf{2}$ of $\mathbf{2 4}$

\section{Abstract}

A Therapeutic Nipple Shield (TNS) was previously developed to respond to the global need for new infant drug delivery technologies. However, the release efficiency for the same Active Pharmaceutical Ingredient (API) from different therapeutic matrices within the TNS formulation has not yet been investigated. To address this, in-vitro release of elemental zinc into human milk from two types of Texel non-woven fibre mats of varying thickness and different gram per square meter values, placed inside the TNS was explored and compared to the release from zinc-containing rapidly disintegrating tablets. In-vitro delivery was performed by means of a breastfeeding simulation apparatus, with human milk flow rates and suction pressure adjusted to physiologically relevant values, and release was quantified using Inductively Coupled Plasma Optical Emission Spectrometry (ICP-OES). It was found that a total recovery of $62-64 \%$ elemental zinc was obtained after the human milk had passed through the fibre insert, amounting to a 20 - 48\% increase compared to previous zinc delivery studies using rapidly disintegrating tablets within the TNS. This indicates that non-woven Texel fibre mats were identified as the superior dosage form for zinc oral drug delivery into human milk using a TNS.

Keywords: pediatrics; oral delivery; breastfeeding; human milk; medication systems; X-ray Micro Computed Tomography 
Page $\mathbf{3}$ of $\mathbf{2 4}$

\section{Graphical Abstract}

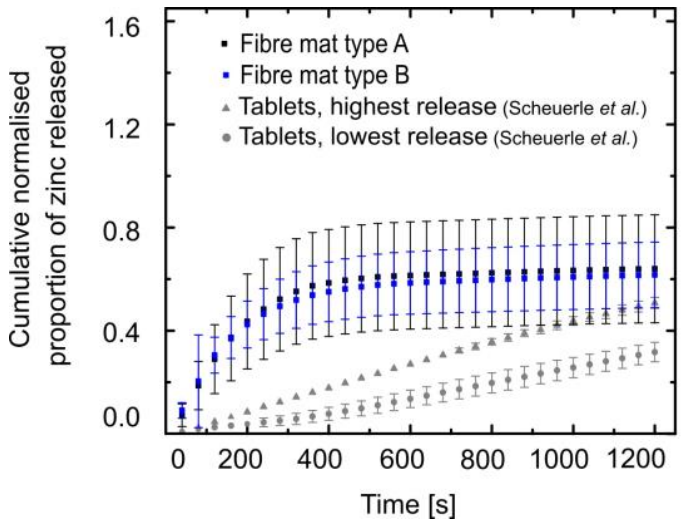

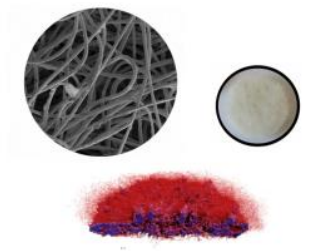

Texel non-woven fibre mat type A loaded with zinc sulfate

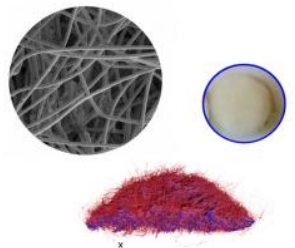

Texel non-woven fibre mat type B loaded with zinc sulfate 
Page $\mathbf{4}$ of $\mathbf{2 4}$

\section{Introduction}

New infant drug delivery technologies are urgently needed. According to the World Health Organization (WHO), many of the 5.9 million child deaths worldwide before the age of five could be prevented by enabling access to affordable interventions, capable of addressing the current challenges of low-resource settings, such as lack of potable water or sterilisation (IGME, 2015; World Health Organization, 2007). This clearly indicates that novel, affordable and easy-to-use infant drug delivery technologies are urgently needed. To address current limitations, a Therapeutic Nipple Shield (TNS) was developed (Gerrard et al., 2012), which enables the delivery of Active Pharmaceutical Ingredients (APIs) during breastfeeding. It consists of a nipple shield device containing a therapeutic insert, for example in the form of an API-loaded tablet or fibrous material. When worn by a mother during breastfeeding, such a device enriches the human milk consumed by the suckling infant with medication or nutrients (see Figure 1) (Gerrard et al., 2012).

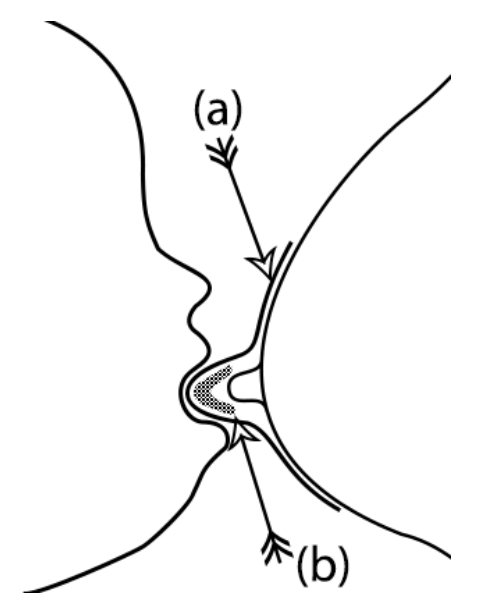

Figure 1: Working principle of the TNS. The TNS is placed on the mother's breast during breastfeeding, while the flow of human milk delivers the therapeutic to the suckling infant. (a) Nipple shield device, (b) Active Pharmaceutical Ingredient (API). 
The TNS has the potential to dramatically change the way drugs are administered to infants worldwide, as it could potentially improve the acceptability of pharmaceutical products in both developed and developing countries (Lopez and Ernest, 2015), and offer an alternative to conventional drug delivery for the $36 \%$ of infants aged $0-6$ months, who are exclusively breastfed (World Health Organization, 2017).

Zinc is a physiologically important element, responsible for the activity of various proteins in major pathways of the human metabolism (Terrin et al., 2015). Its deficiency has extensive effects on growth and development of infants, as well as the capabilities of the body's immune system (Caulfield et al., 1998; Terrin et al., 2015; Wulf et al., 2013). The intestinal absorption of zinc is independent of zinc status, yet higher uptake is reported for term babies as a result of lower zinc concentration in the blood serum (Terrin et al., 2015). Preterm and Small for Gestational Age (SGA) neonates have an increased risk of zinc deficiency (Wulf et al., 2013), which is believed to occur within the first 1 - 2 months of life (Caulfield et al., 1998). Breastfeeding infants obtain zinc through the consumption of human milk, however, zinc concentration can exhibit significant variations $\left(0.7-1.6 \mathrm{mg} \mathrm{l}^{-1}\right)$, and constantly declines postpartum:

$8-12 \mathrm{mg}^{-1}$ zinc can be found in colostrum, the first milk the maternal breast produces after giving birth, 3 - $6 \mathrm{mg}^{-1}$ in human milk seven days after birth, and only 1- $3 \mathrm{mg}^{-1}$ an additional three weeks later (Terrin et al., 2015). To prevent deficiency, enteral delivery of zinc is recommended, being $0.8 \mathrm{mg} \mathrm{kg}^{-1} \mathrm{day}^{-1}$ for term infants and $3 \mathrm{mg} \mathrm{kg}^{-1}$ day $^{-1}$ for preterm neonates (Hambidge and Krebs, 2004). If zinc deficiency occurs, it is associated with conditions such as dermatitis, growth retardation, necrotizing enterocolitis, neurologic damage, bronchopulmonary dysplasia, infections, and retinopathy of prematurity (Terrin et al., 2015). It is recognized that not only severe, but also moderate zinc deficiency is adding to "the total burden of disease" in infants leading to about 800,000 child deaths every year (Black, 2003). In addition to zinc's general health promoting effects, the WHO recommends use of zinc 
Page $\mathbf{6}$ of $\mathbf{2 4}$

(20 mg zinc sulphate) as an adjunct supplement to oral rehydration salts for the treatment of acute diarrhoea (World Health Organization, 2015). Due to the significance of zinc for the physical development and wellbeing of infants globally, its delivery into human milk using the TNS was investigated in this study.

To aid in-vitro testing of the TNS, a breastfeeding simulation apparatus was developed by Gerrard et al., simulating both the process of lactation and infant feeding by mimicking the average flow rates/patterns of milk during breastfeeding and the infant's suckling pressure (Gerrard et al., 2013). The apparatus was previously used to investigate release of a Sulforhodamine B dye and of zinc sulphate from rapidly disintegrating tablets into human milk (Gerrard et al., 2012; Scheuerle et al., 2017). The capability of non-woven fibres to release APIs into human milk was previously shown by Gerrard et al., who investigated the delivery of Sodium Dodecyl Sulphate (SDS) from loaded non-woven fibres (Bathfelt, Texel, Québec, Canada) located within a Sinnex filter holder, but without the use of breastfeeding simulation (Gerrard et al., 2012). The following research builds on both studies and explored the fibrebased zinc sulphate delivery from a nipple shield device into human milk using the breastfeeding simulation apparatus. It is the first time that absolute recovery of the same compound using different dosage forms within the TNS has been quantitatively compared. 
Page $\mathbf{7}$ of $\mathbf{2 4}$

\section{Materials and methods}

\subsection{Materials}

In accordance with previous fibre studies by Gerrard et al. (Gerrard et al., 2012), non-woven fibre mats (35\% viscose, 65\% polyester), provided from Bathfelt, Texel (Québec, Canada) were used as a delivery matrix. Two different types of fibre mats, placed within the same lipcontaining nipple shield device as previously applied for the evaluation of zinc delivery via rapidly disintegrating tablets by Scheuerle et al. (Scheuerle et al., 2017), were investigated: a 235 gram per square meter $\left(\mathrm{g} \mathrm{m}^{-2}\right)$ felt with targeted thickness of $1.8 \mathrm{~mm}$ (referred to as fibre mat type A), and a $335 \mathrm{~g} \mathrm{~m}^{-2}$ felt with targeted thickness of $2.1 \mathrm{~mm}$ (referred to as fibre mat type B). An illustration can be found in Figure 2. Zinc sulphate monohydrate was obtained from Sigma Aldrich (Dorset, UK), and preferred over elemental zinc based on its superior solubility and taste characteristics (Scheuerle et al., 2017). Human milk samples were supplied by the Queen Charlotte's and Chelsea Hospital Milk Bank (Imperial College Healthcare NHS Trust), and characterized as outlined in previous literature (Scheuerle et al., 2017), except that the fat content was calculated using an updated creamatocrit to fat correlation, published by Mitoulas et al. (Mitoulas et al., 2002). Human milk used for experiments in this study had a protein content of $43.52 \mathrm{~g} \mathrm{l}^{-1}$, and a fat content of $16.51 \mathrm{~g} \mathrm{l}^{-1}$. Both values correspond to previously reported literature data (Emmett and Rogers, 1997; Kent et al., 2006; Saarela et al., 2005). The Cambridge Human Biology Research Ethics Committee at the University of Cambridge approved all human milk sample use. 
a)

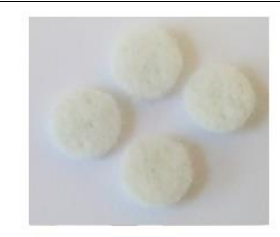

c)

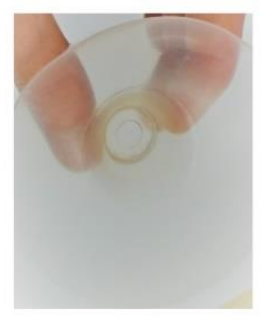

b)

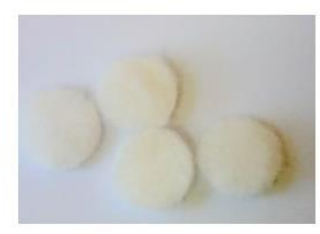

d)

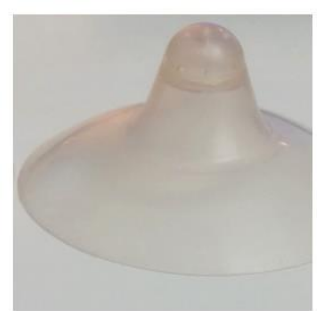

Figure 2: Fibres and nipple shield device used in the study. a - b) Illustration of non-woven fibre mat (a) type A and (b) type B, loaded with zinc sulphate. c - d) Lip-containing nipple shield device used for all breastfeeding simulation experiments.

\subsection{Methods}

\subsubsection{Loading procedure}

$6.2 \mathrm{mg}$ (standard deviation $0.075 \mathrm{mg}, \mathrm{n}=3$ ) and $6.4 \mathrm{mg}$ (standard deviation $0.046 \mathrm{mg}, \mathrm{n}=3$ ) of elemental zinc was loaded through administration of a $49 \%$ zinc sulphate monohydrate solution (wt/wt \%) onto type A and B fibre mats, respectively. Values were based on the WHO dose recommendation of zinc sulphate as an adjunct to oral rehydration salts for the treatment of acute diarrhoea (World Health Organization, 2015). All fibre mats were air dried in a petri dish for 24 hours at room temperature.

\subsubsection{Scanning Electron Microscope analysis}

Fibre mat cut-outs (diameter $10 \mathrm{~mm}$ ), unloaded as well as loaded with zinc sulphate monohydrate respectively, were mounted on $12.5 \mathrm{~mm}$ Cambridge stubs with Silver Dag (Taab Ltd), sputter coated with $15 \mathrm{~nm}$ of iridium with a Quorum/Emitech K575X sputter coater, and viewed using a FEI Verios 460L Scanning Electron Microscope at $3 \mathrm{kV}$ and $25 \mathrm{pA}$ using a through lens detector operated in field free mode. 


\subsubsection{X-ray Micro Computed Tomography}

X-ray Micro Computed Tomography $(\mathrm{X} \mu \mathrm{CT})$ of the entire fibre mat was performed using a SkyScan 1172 high-resolution X $\mu \mathrm{CT}$ scanner (Bruker, Antwerp, Belgium), combining a cone beam geometry and a $2 \mathrm{D}$ array detector. By rotation of the object over $180^{\circ}$ in steps of $0.25^{\circ}$, shadow projections are acquired, from which a 3D image can be obtained following tomographic reconstruction. This imaging method was chosen due to its high spatial resolution and negligible diffraction in the investigated samples. Imaging was performed at an isotropic voxel size of $2.49 \mu \mathrm{m}, 10$ images per position were averaged to reduce noise and increase the contrast. The total acquisition time per sample amounted to approximately $3 \mathrm{~h}$, in which 720 images were recorded. Data obtained was reconstructed using NRecon (Bruker, v1.6.8.0), visualized in CTVox (Bruker, v3.3), and processed in Avizo Fire (FEI Company, Hillsboro, Oregon, USA, v8.1). Thresholding was applied to separate the solid material from the pore space for a subvolume $\left(0.998 \mathrm{~mm}^{3}\right)$ of the loaded and unloaded fibre mat. The volume and surface were calculated for the extracted solid material and the porosity was quantified by relating the volume of the pore space to that of the solid material.

\subsubsection{Delivery and quantification}

Calibration of the breastfeeding simulation apparatus' pumps prior to any experimental work being undertaken, as well as monitoring of temperature and pressure throughout the breastfeeding simulation was performed. Delivery of zinc was conducted in triplicate for each type of fibre mat by means of the breastfeeding simulation apparatus (Gerrard et al., 2013). Based on literature about breastfeeding physiology (Black et al., 1998; Geddes et al., 2008; Macias and Meneses, 2011; Moral et al., 2010), and in accordance with previous work conducted using the simulation apparatus (Gerrard and Larson, 2013; Scheuerle et al., 2017), a

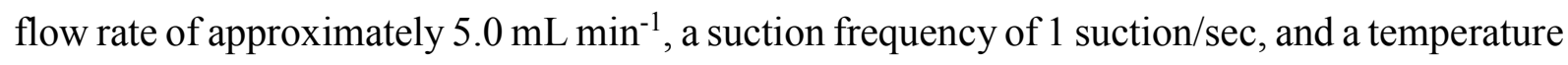
range of $33.4-33.7^{\circ} \mathrm{C}$, simulating the likely temperature of human milk in the infant's mouth, 
were chosen for all experiments. The pressure range and amplitude were adjusted to values of physiological relevance (Geddes et al., 2008). Peak vacuum values of $-22.78 \mathrm{kPa}$ and -22.32 $\mathrm{kPa}$ as well as baseline vacuum values of $-10.90 \mathrm{kPa}$ and $-11.38 \mathrm{kPa}$ for fibre mat type $\mathrm{A}$ and B respectively were recorded. Figure 3 illustrates an exemplary average pressure profile for each type of fibre mat. All experiments were conducted in triplicate for each type of mat. The human breast mimic was set to an angle of $30^{\circ}$ downwards from the vertical axis. Fractions were collected for a period of $40 \mathrm{~s}$ each with a total of 30 fractions, amounting to a total of 20 min. This duration corresponds to double the time of an average breastfeed (Geddes et al., 2008), and was chosen based on previously published breastfeeding simulation by Scheuerle et al. to allow for comparative analysis (Scheuerle et al., 2017). All samples collected by the fraction collector were weighted using a Sartorius analytic balance (Epsom, UK), and subsequently stored at $-80^{\circ} \mathrm{C}$ until further analysis.

a)

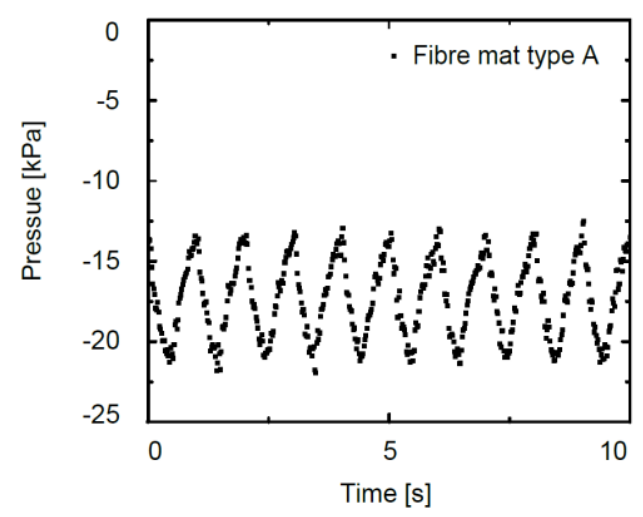

b)

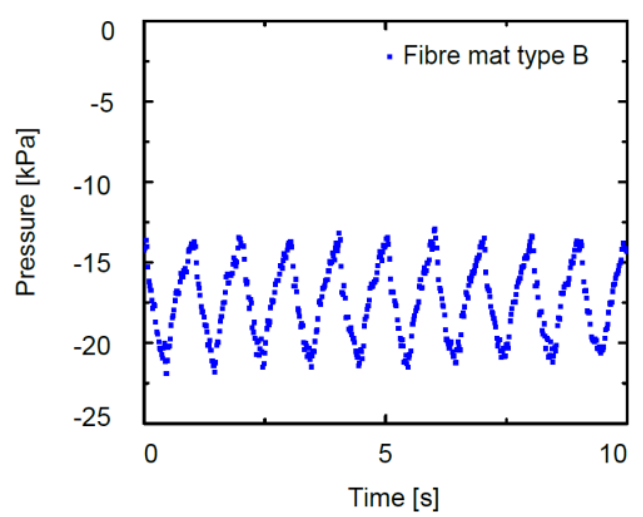

Figure 3: Example pressure profiles during zinc delivery experiments using the breastfeeding simulation apparatus, depicted for $10 \mathrm{~s}$ at $\mathrm{t}=120-130 \mathrm{~s}$. Dosage forms used for the TNS were zinc-loaded fibre mats a) type A, and b) type B. Experiments were conducted using an average

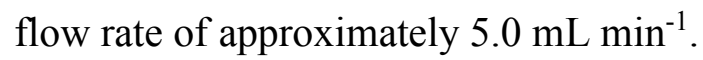


Both the overall amount of zinc loaded onto the fibre mats used for experimental procedures, as well as the zinc content in collected fractions during breastfeeding simulation, were evaluated using Inductively Coupled Plasma Optical Emission Spectrometry (ICP-OES) at the MRC Human Nutrition Research Unit (Cambridge, UK). Zinc detection in nine out of the 30 human milk samples was performed according to a protocol by Scheuerle et al., while the zinc concentration within the unmeasured human milk samples was subsequently evaluated by means of linear interpolation (Scheuerle et al., 2017). The amount of elemental zinc loaded onto the non-woven fibre mats was evaluated as follows: Fibre mats were added to a vial containing $1 \mathrm{~mL}$ rinse of ultra-high purity water with $0.002 \%$ TritonX100. Following two 10 -minute rounds of ultra-sonication, all vials were placed on a shaker plate for gentle shaking at night, and $1 \mathrm{~mL}$ of rinse was added during the day. This process was performed for a total of three nights/days, following which the samples were held in a $40^{\circ} \mathrm{C}$ water bath over night. After a 1:10 volumetric dilution in ultra-purity water, samples were diluted 1:20 volumetrically in a diluent, consisting of ultra-high purity water (Sartorius Stedium Biotech Arium pro UV Water polisher) with $0.002 \%$ TritonX100 (Sigma-Aldrich, UK) and a standard of 1 ppm molybdenum (SPEX CertiPrep Metuchen, NJ). All sample solutions were vortexed using a Fisherbrand Whirlimixer (Loughborough, UK), and subsequently analysed according to the ICP-OES protocol as described by Scheuerle et al. (Scheuerle et al., 2017). Data obtained was used to generate a release profile and to evaluate the cumulative zinc release.

\section{Results and discussion}

\subsection{Scanning Electron Microscope analysis}

SEM images of unloaded and zinc sulphate loaded Texel fibre mats type A and type B were obtained to explore the fibre mats morphology (see Figure 4 and Figure 5). It was found that despite their difference in $\mathrm{g} \mathrm{m}^{-2}$ and thickness, both fibre mat types have the same fibrous structure and an approximate fibre diameter of $20 \mu \mathrm{m}$. Zinc sulphate was identified qualitatively 
by morphological analysis, indicating its adsorption in localized areas scattered across the fibrous mat network, either in form of flat patches or clumps. It forms a cracked, but mostly uniform coating on the fibre surface, caused by the evaporation of water from the loaded zinc sulphate solution. Due to the porous structure, adsorption within the deeper regions of the fibre mats can be observed.
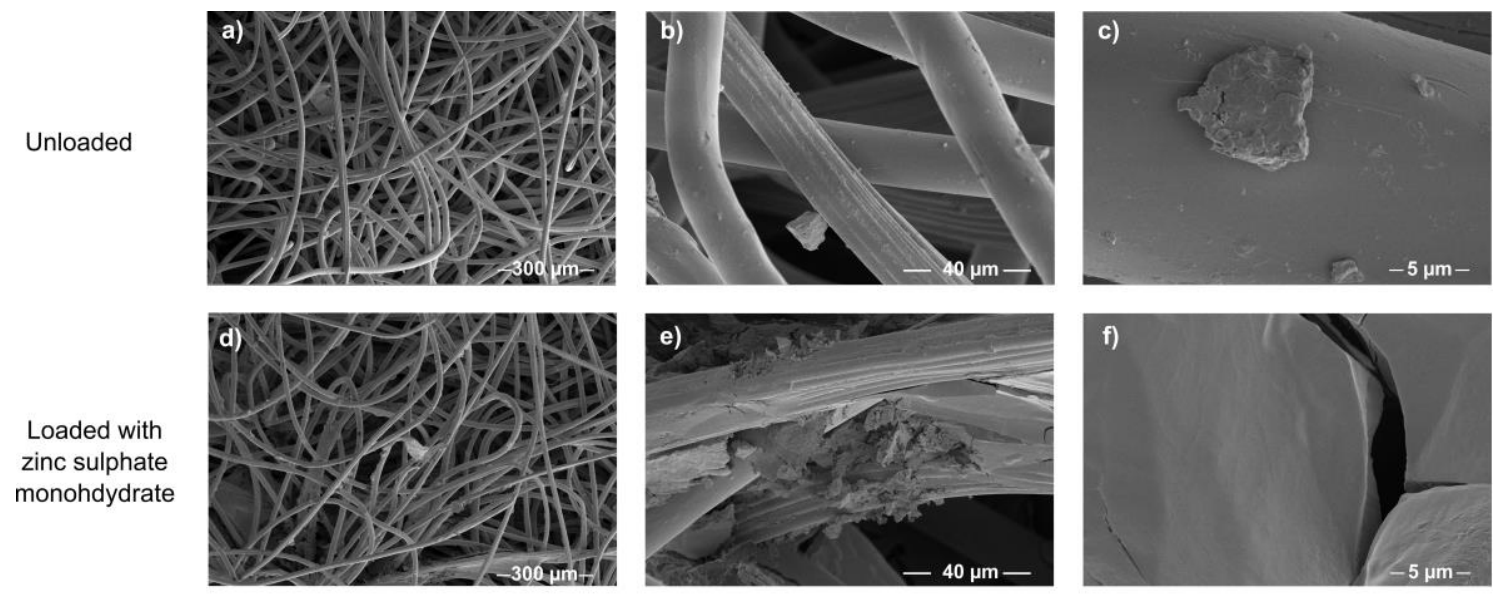

Figure 4: SEM results for fibre mat type A. SEM images illustrating the surface of Texel fibre mat type A (1.8 $\mathrm{mm}$ targeted thickness, $\left.225 \mathrm{~g} \mathrm{~m}^{-2}\right)$ at different magnifications. Images a) - c) present unloaded fibre mats, images, d) - f) fibre mats loaded with zinc sulphate.
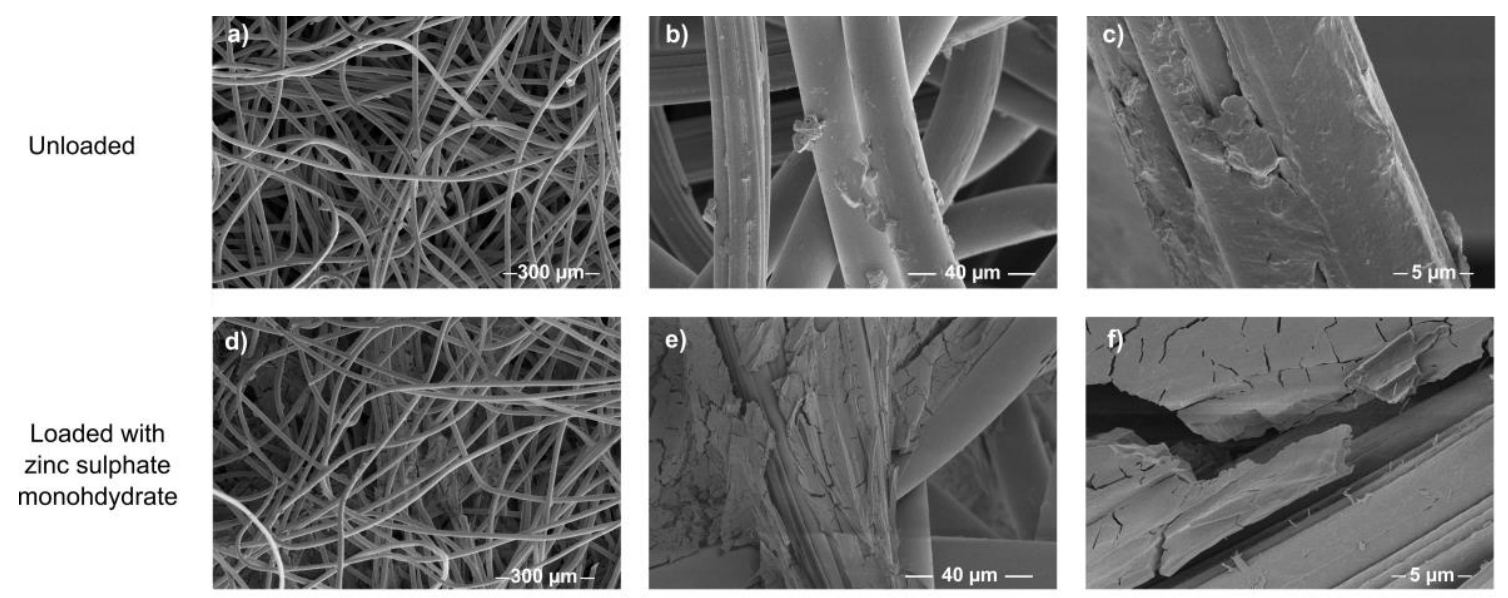

Figure 5: SEM results for fibre mat type B. Illustration of results obtained for SEM analysis of Texel fibre mat type B (2.1 mm targeted thickness, $\left.325 \mathrm{~g} \mathrm{~m}^{-2}\right)$ at different magnifications. Images a) - c) present unloaded fibre mats, images d) - f) fibre mats loaded with zinc sulphate. 
Page $\mathbf{1 3}$ of $\mathbf{2 4}$

\subsection{X-ray Micro Computed Tomography}

X-ray Micro Computed Tomography $(\mathrm{X} \mu \mathrm{CT})$ was used to investigate the fibre mats' porosity and 3D structure. Figure 6 and Figure 7 illustrate loaded and unloaded Texel fibre mats type A and type B, respectively. Different colours refer to material of different density, red indicating low and green high density material. Based on the density values of viscose $\left(1.52 \mathrm{~g} \mathrm{~cm}^{-3}\right)$ (Koc and Cincik, 2013), polyester (1.38 $\mathrm{g} \mathrm{cm}^{-3}$ ) (Koc and Cincik, 2013), and zinc sulphate (3.54 g $\mathrm{cm}^{-3}$ ) (FisherScientific, 2014), the presented colour scale was chosen to illustrate unloaded fibre material of comparably lower density in red, and zinc sulphate of comparably higher density in blue and green colour. While Figure 6 and Figure 7 depict varying amounts of fibre material in images d) - e), image f) solely displays loaded zinc sulphate. This becomes particularly apparent in direct comparison with the unloaded fibre mats in images a) - c), in which the visible fibrous material at medium density can only barely be visible as a faint silhouette, with mostly light blue spots of $<10 \mu \mathrm{m}$ and sporadically larger spots of $<250 \mu \mathrm{m}$ size visible. The bottom side of fibre mat type A, unlike fibre mat type B exhibits a fibrous structure similar in appearance to those of unloaded fibre mats (see Figure 8). In agreement with the SEM analysis we found that the diameter of individual fibres was approximately $20 \mu \mathrm{m}$ for unloaded or partially loaded fibres. Loading with zinc sulphate appears to result in swelling of individual fibres as well as fusing of adjoined fibres where they physically touch or where they are sufficiently close to come in contact during swelling. As a result, individual fibres are no longer identifiable in areas of high zinc loading. Figure 9 shows examples of representative cross-sectional cuts through a swollen fibre cluster. The volume fraction, porosity, and total surface area were calculated for a cubical region of interest measuring $0.998 \mathrm{~mm}^{3}$ from the fibre mats' centre (Table 1). 

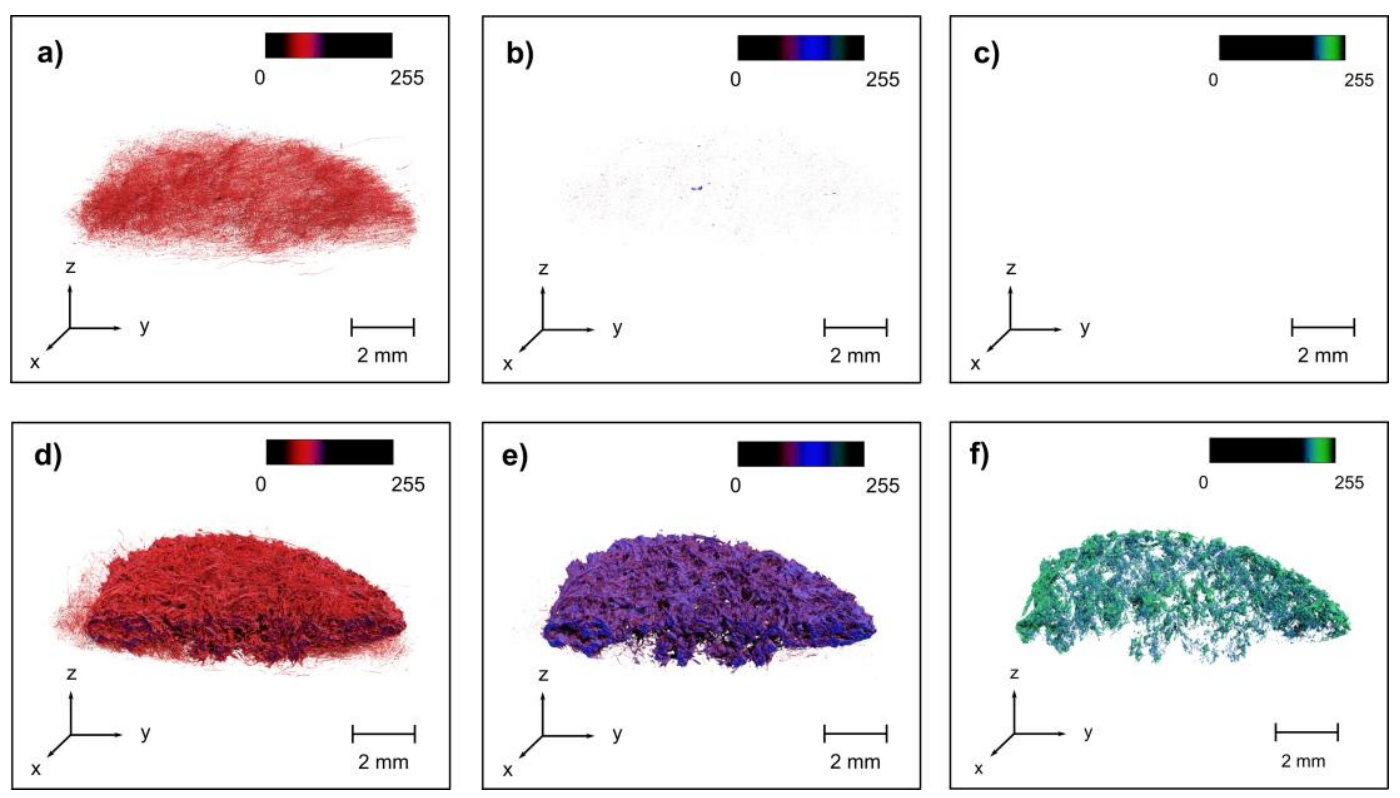

Figure 6: Images generated from $\mathrm{X} \mu \mathrm{CT}$ measurements of a) - c) unloaded, and d) - f) zinc sulphate loaded Texel fibre mat type A (1.8 $\mathrm{mm}$ targeted thickness, $\left.225 \mathrm{~g} \mathrm{~m}^{-2}\right)$. The colour maps were the same for all images and only the opacity was adjusted in order to visualize components of different densities. Red colour indicates low, green high density material. The images depict only half of the fibre mats in order to visualize the cross-section at the centre of each sample.
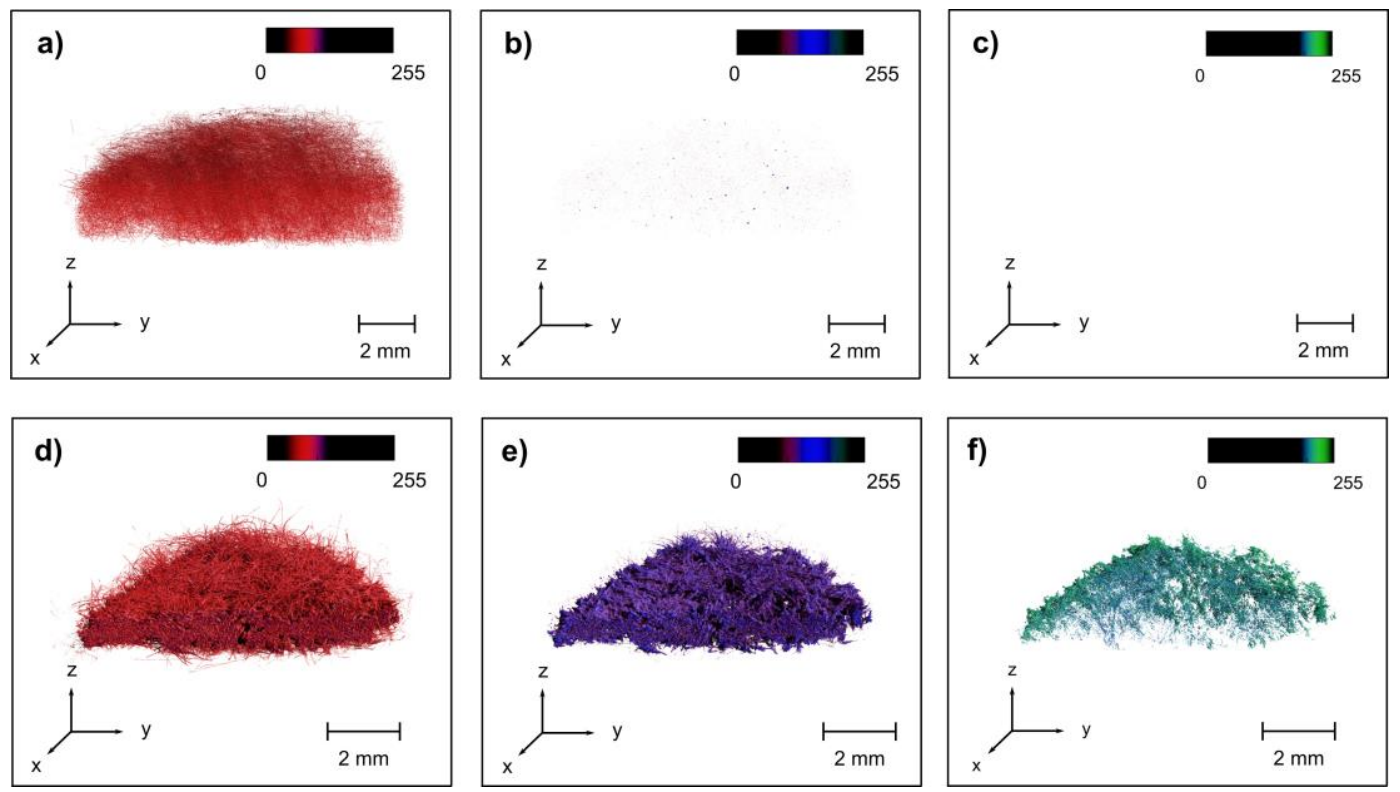

Figure 7: Images generated by $\mathrm{X} \mu \mathrm{CT}$ analysis of a) - c) unloaded, and d) - f) zinc sulphate loaded Texel fibre mat type B (2.1 mm targeted thickness, $\left.325 \mathrm{~g} \mathrm{~m}^{-2}\right)$. 


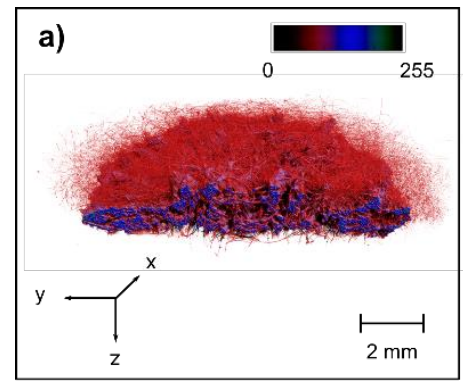

Figure 8: Images generated for $\mathrm{X} \mu \mathrm{CT}$ analysis of loaded Texel fibre mats a) type A, b) type B (bottom side facing up).

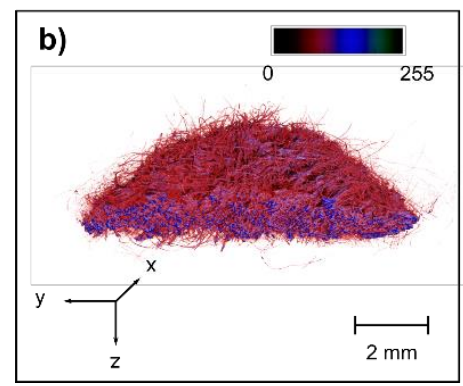

\begin{tabular}{|c|c|c|c|c|}
\hline & Parameter & Unloaded & Loaded & Change [\%] \\
\hline \multirow{3}{*}{ Fibre mat type A } & Porosity [-] & 0.942 & 0.607 & -36 \\
\hline & Surface area $\left[\mathrm{mm}^{2}\right]$ & 19.72 & 32.70 & +66 \\
\hline & Volume $\left[\mathrm{mm}^{3}\right]$ & 0.058 & 0.392 & +580 \\
\hline \multirow{3}{*}{ Fibre mat type B } & Porosity [-] & 0.939 & 0.416 & -56 \\
\hline & Surface area $\left[\mathrm{mm}^{2}\right]$ & 17.30 & 50.90 & +194 \\
\hline & Volume $\left[\mathrm{mm}^{3}\right]$ & 0.061 & 0.583 & +856 \\
\hline
\end{tabular}

Table 1: Overview of porosity, surface area, and volume of a cubical region of interest measuring $0.998 \mathrm{~mm}^{3}$ taken from the loaded and unloaded fibre mats' centre. 

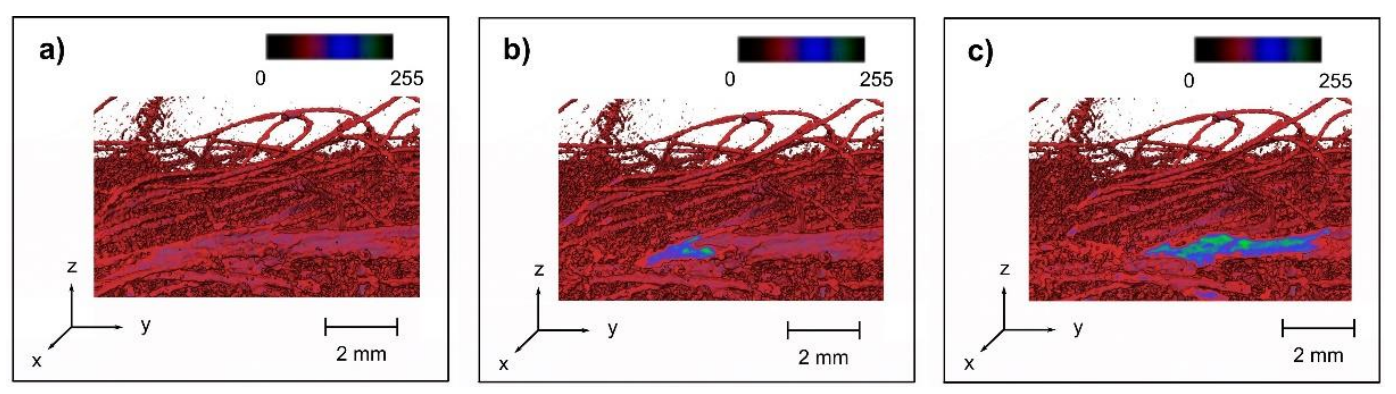

Figure 9: Cut through zinc-loaded fibres in the y-z plane. Images a) - c) illustrate the resulting cross-sectional images at different position in the $\mathrm{x}$-direction. Zinc-loaded fibres appear swollen and fused with adjoined fibres to form high-density fibre clusters.

\subsection{Release of zinc from non-woven fibres}

The average total elemental zinc content loaded onto the fibre mat amounted to $6.17 \pm 0.27 \mathrm{mg}$ for fibre mat type $\mathrm{A}$, and $6.4 \pm 0.05 \mathrm{mg}$ for fibre mat type B. The cumulative normalized proportion of zinc released and the normalized proportion of zinc released in individual fractions over time are illustrated in Figure 10. The amount of human milk passed through the TNS, as well as the absolute recovery achieved for both fibre mat types are presented in Table 2. Effectiveness and duration of release for both fibre mat types were comparable. 
a)

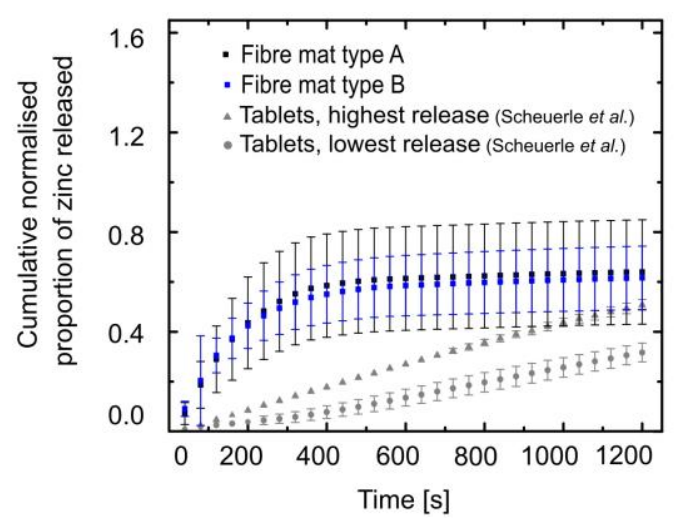

b)

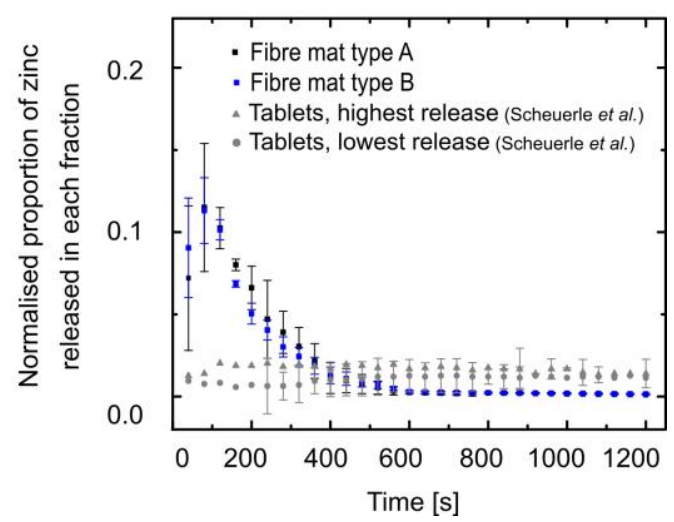

Figure 10: Release of zinc sulphate from Texel fibre mats type A (1.8 mm targeted thickness, $\left.235 \mathrm{~g} \mathrm{~m}^{-2}\right)$ and type $\mathrm{B}\left(2.1 \mathrm{~mm}\right.$ targeted thickness, $\left.335 \mathrm{~g} \mathrm{~m}^{-2}\right)$, as well as from rapidly disintegrating tablets by Scheuerle et al. (Scheuerle et al., 2017) into human milk using the breastfeeding simulation apparatus by Gerrard et al. (Gerrard et al., 2012). Experiments were conducted at $33.7-35.7^{\circ} \mathrm{C}$, and a mean flow rate of approximately $5.0 \mathrm{~mL} \mathrm{~min}^{-1}$ was applied. a) Cumulative normalized proportion of zinc sulphate released and b) Normalized proportion of zinc sulphate released in each fraction over time. Release is represented as a percentage of the total amount loaded onto the non-woven fibre mats to the total amount released. Each set of points represents the average of three experiments, using the mean of ICP-OES triplicate measurements for each fraction shown.

\begin{tabular}{|l|c|c|}
\hline & Recovery achieved [\%] & $\begin{array}{l}\text { Amount of human milk } \\
\text { passed through the device }[\mathrm{g}]\end{array}$ \\
\hline Mat type A & $64.00 \pm 0.01$ & $93.09 \pm 1.08$ \\
\hline Mat type B & $61.64 \pm 0.01$ & $98.15 \pm 0.97$ \\
\hline
\end{tabular}

Table 2: Recovery achieved and human milk passed through the TNS for the delivery of zinc from Texel non-woven fibres using the breastfeeding simulation apparatus within the duration of 20 minutes (fractions of $40 \mathrm{~s}$ each with a total of 30 fractions). 
Full delivery of zinc into human milk within the duration of one breastfeed, as previously demonstrated to amount to $76.0 \pm 12.6 \mathrm{~g}$ of human milk for 1-month- to 6-months-old infants (Kent et al., 2006), has not been achieved. This could potentially be caused by milk components accumulating within the porous mat structure, thereby hampering the flow of milk through, and thus the delivery of zinc from the fibre network. The nature and absorption properties of the API significantly affect the release rate, however recent research appears to suggest that the lipid content and distribution within the milk influences the absolute recovery: Previous studies of SDS delivery from Texel non-woven fibres within a Sinnex filter holder into cow's milk have shown that absolute release was decreased to $30-60 \%$ when using non-homogenized milk (5.2\% fat) as opposed to $70-90 \%$ for use of an homogenized form (3.6 \% fat) (Gerrard et al., 2012). SDS release of approximately $100 \%$ into non-homogenized goat's milk (4.0\% fat) however, made Gerrard et al. conclude that in addition to the concentration and distribution of lipids, the overall milk composition, including carbohydrates, proteins, and lipids, has an impact on the API release rate from Texel non-woven fibres (Gerrard et al., 2012). Both observations present a challenge for the development of a standardized protocol for therapeutic release via non-woven Texel fibre mats, as human milk is a complex fluid with highly variable properties. To give but one example, absolute fat content of human milk can vary up to three fold, depending on the stage of lactation, dietary requirements and health status of the breastfeeding mother (Vir, 2011). Potential surface modifications, such as plasma treatment, use of the wet chemical method, or graft polymerization of the fibres' surface (Ikada, 1994; Yoo et al., 2009), could provide a means to address unwanted milk components accumulation while enhancing adsorption properties, yet thereby also increasing manufacturing effort and cost of production. Moreover, the obtained $\mathrm{X} \mu \mathrm{CT}$ results also suggest that changes in fibre conformation are likely impacting the release properties of the Texel fibre mats. Given the decrease in porosity as a result of the zinc loading process, flow of human milk through the fibrous structure is restricted following loading, and can further be impacted by the 
accumulation of milk components. Previously, it was reported that the release from low surface area to volume hydroxypropylmethylcellulose tablets is slower (Reynolds et al., 2002), and similarly, based on literature by Siepmann et al. (Siepmann and Siepmann, 2012), the release from zinc-loaded fibre clusters with their reduced surface to volume ratio is believed to be slowed down. The $\mathrm{X} \mu \mathrm{CT}$ data of unloaded fibre mats revealed the presence of fibres of higher density than originally expected based on the densities of viscose and polyester. In addition, fibre accumulation and/or the presence of impurities can be observed as indicated by the $10-250 \mu \mathrm{m}$ sized spots that are visible at medium density values in the $\mathrm{X} \mu \mathrm{CT}$ data, believed to be a result of the fibres' manufacturing process, and/or caused by trapped dust particles. The even distribution of zinc within the fibre mat network of fibre mat type B in contrast to mat type A (Figure 8) is attributed to the larger fibre diameter and different wetting behaviour of the two fibre mats. The liquid absorption of the fibre mats as well as of the individual fibres is driven by capillary action and strongly influenced by the porosity of the samples (Chen et al., 1995). It would also explain the unequal changes in porosity, surface area, and volume of fibre mat type A and B following loading, which however do not lead to an observed difference in release from both mats.

Overall, the absolute release of zinc from Texel non-woven fibres is $20-48 \%$ superior compared to rapidly disintegrating tablets manufactured by direct compression: In a study by Scheuerle et al. achieved absolute recovery amounted to only 32 - 51\% (Scheuerle et al., 2017). It has to be noted that the total elemental zinc content of the rapidly disintegrating tablets (17.81 -18.21 mg) was higher than the amount of elemental zinc loaded onto the Texel nonwoven fibre mats used in this study (6.17-6.40 mg) (Scheuerle et al., 2017). Yet, while the main release from non-woven fibres occurred within the first $8 \mathrm{~min}$, the release profile using rapidly disintegrating tables was linear, meaning that after 8 min the estimated elemental zinc content ranged between 2 - $4 \mathrm{mg}$ (Scheuerle et al., 2017). This was significantly lower than the release from Texel non-woven fibre mats at $8 \mathrm{~min}$. In addition to their superior release properties, the 
Page $\mathbf{2 0}$ of $\mathbf{2 4}$

tested Texel non-woven fibre mats are characterized by enhanced loading feasibility and costeffectiveness compared to the referenced rapidly disintegrating tablets, supporting their use as a dosage form of the TNS.

Important factors to be taken into consideration prior to in-vivo studies are fibre cohesion in order to exclude the possibility of fibre break-off, palatability, biocompatibility, and long-time stability. Moreover, use of different fibre materials, and different fibre types, such as fastdisintegrating electrospun fibres fabricated into non-woven film, could be investigated in future research.

\section{Conclusions}

Using a breastfeeding simulation apparatus to mimic the process of lactation and infant feeding, delivery of zinc sulphate from non-woven Texel fibre mats into human milk was investigated. With an absolute elemental zinc recovery of $64 \%$ (fibre mat type A) and 62\% (fibre mat type B) respectively in $93 \mathrm{~g}$ and $98 \mathrm{~g}$ of human milk passed through the fibre insert, full delivery within one breastfeed (approximately $76 \mathrm{~g}$ ) has not been achieved. This can be attributed to the complex properties of human milk and accumulation of milk components within the porous mat structures, as well as structural changes of the fibre networks following loading, thereby hampering the delivery of zinc. In comparison to previous experiments however, the absolute zinc recovery using non-woven Texel fibre mats was identified as superior to the release by means of rapidly disintegrating tablets (Scheuerle et al., 2017). Based on the non-woven fibre mats' release characteristics and cost-effectiveness, non-woven fibres can be regarded as an advantageous dosage form for oral drug delivery using the TNS. Further research will be required to establish Texel non-woven fibres as a generalized matrix for oral drug delivery, and

to further explore alternative fibrous materials with advantageous characteristics for API delivery into human milk. 
Page $\mathbf{2 1}$ of $\mathbf{2 4}$

\section{Acknowledgements}

This work was supported by the United States Agency for International Development (USAID), the Government of Norway, the Bill \& Melinda Gates Foundation, Grand Challenges Canada and the UK Department for International Development (DFID) through the Saving Lives at Birth Award Scheme [grant number 0454-03]. Theresa Maier (T.M.) was supported through academic scholarships by the WD Armstrong Trust, University of Cambridge, and the German National Merit Foundation, Rebekah Scheuerle (R.L.S.) through a Gates Cambridge Trust scholarship, and Sylvaine Bruggraber (S.F.A.B.) through the Medical Research Council [grant number MC_US_A090_0008/Unit Program number U1059]. We thank Gillian Weaver, manager of the Queen Charlotte's and Chelsea Hospital Milk Bank (Imperial College Healthcare NHS Trust) for enabling this study by providing human milk samples, Jeremy Skepper (Cambridge Advanced Imaging Centre) for conducting SEM imaging, and Stephen Gerrard for having previously built the breastfeeding simulation apparatus and developed the NDSD nipple shield design used in this study.

\section{Competing interests}

Co-author Rebekah Scheuerle is an inventor of three provisional patents related to the previous invention of the NSDS nipple shield design (US patent applications: No. 62/307,375, $62 / 337,805$, and 62/424,006, all of which are called "Device and Method for Delivering an Agent into Breast Milk while Breastfeeding"). This does not affect the adherence to policies of the International Journal of Pharmaceutics. 
Page $\mathbf{2 2}$ of $\mathbf{2 4}$

\section{References}

Black, R.E., 2003. Zinc deficiency, infectious disease and mortality in the developing world. J. Nutr. 133, 1485-1489.

Black, R.F., Jarman, L., Simpson, J., 1998. The Science of Breastfeeding, 3rd ed. Jones and Bartlett Publishers.

Caulfield, L.E., Zavaleta, N., Shankar, A.H., 1998. Potential contribution of maternal zinc supplementation during pregnancy to maternal and child survival. Am. J. Clin. Nutr. 68, 499S-508S.

Chen, Y.-T., Davis, H.T., Macosko, C.W., 1995. Wetting of fiber mats for composites manufacturing: I. Visualization experiments. AIChE J. 41, 2261-2273. doi:10.1002/aic.690411009

Emmett, P.M., Rogers, I.S., 1997. Properties of human milk and their relationship with maternal nutrition. Early Hum. Dev. 49, S7-S28. doi:10.1016/S0378-3782(97)00051-0

FisherScientific, 2014. Safety Data Sheet - Zinc Sulfate. FisherScientific.

Geddes, D.T., Kent, J.C., Mitoulas, L.R., Hartmann, P.E., 2008. Tongue movement and intraoral vacuum in breastfeeding infants. Early Hum. Dev. 84, 471-477. doi:10.1016/j.earlhumdev.2007.12.008

Gerrard, S.E., Baniecki, M.L., Sokal, D.C., 2012. A nipple shield delivery system for oral drug delivery to breastfeeding infants: Microbicide delivery to inactivate HIV. Int. J. Pharm. 434, 224-234. doi:10.1016/j.ijpharm.2012.05.035

Gerrard, S.E., Larson, A.M., 2013. Reducing infectivity of HIV upon exposure to surfaces coated with N, N-dodecyl, methyl-polyethylenimine. Biotechnol. Bioeng. 110, 20582062. 
Gerrard, S.E., Orlu-Gul, M., Tuleu, C., Slater, N.K.H., 2013. Modeling the physiological factors that affect drug delivery from a nipple shield delivery system to breastfeeding infants. J. Pharm. Sci. 102, 3773-3783. doi:10.1002/jps.23688

Hambidge, K.M., Krebs, N.F., 2004. Fetal and Neonatal Physiology.

IGME, U., 2015. Levels and Trends in Child Mortality: Report 2015.

Ikada, Y., 1994. Surface modification of polymers for medical applications. Biomaterials 15, $725-736$.

Kent, J.C., Mitoulas, L.R., Cregan, M.D., Ramsay, D.T., Doherty, D.A., Hartmann, P.E., 2006. Volume and frequency of breastfeedings and fat content of breast milk throughout the day. Pediatrics 117, 387-395. doi:10.1542/peds.2005-1417

Koc, E., Cincik, E., 2013. An analysis on abrasion resistance of polyester-/viscose-blended needle-punched nonwovens. J. Text. Inst. 104, 852-860.

Lopez, F.L., Ernest, T.B., 2015. Formulation approaches to pediatric oral drug delivery: benefits and limitations of current platforms. Expert Opin. Drug Deliv. 12, 1727-1740. doi:10.1517/17425247.2015.1060218

Macias, M.E.R., Meneses, G.J.S.M., 2011. Physiology of nutritive sucking in newborns and infants. Bol. Med. Hosp. Infant. Mex. 68, 296-303.

Mitoulas, L.R., Lai, C.T., Gurrin, L.C., Larsson, M., Hartmann, P.E., 2002. Efficacy of Breast Milk Expression Using an Electric Breast Pump. J. Hum. Lact. 18, 344-352.

Moral, A., Bolibar, I., Seguranyes, G., Ustrell, J.M., Sebastiá, G., Martínez-Barba, C., Ríos, J., 2010. Mechanics of sucking: comparison between bottle feeding and breastfeeding. BMC Pediatr. 10, 1-8. doi:10.1186/1471-2431-10-6

Reynolds, T.D., Mitchell, S.A., Balwinski, K.M., 2002. Investigation of the effect of tablet 
surface area/volume on drug release from hydroxypropylmethylcellulose controlledrelease matrix tablets. Drug Dev. 28, 457-466.

Saarela, T., Kokkonen, J., Koivisto, M., 2005. Macronutrient and energy contents of human milk fractions during the first six months of lactation. Acta Paediatr. 94, 1176-1181. doi:10.1111/j.1651-2227.2005.tb02070.x

Scheuerle, R.L., Bruggraber, S.F.A., Gerrard, S.E., Kendall, R.A., Tuleu, C., Slater, N.K.H., 2017. Characterisation of zinc delivery from a nipple shield delivery system using a breastfeeding simulation apparatus. PLoS One 12, e0171624. doi:10.1371/journal.pone.0171624

Siepmann, J., Siepmann, F., 2012. Modeling of diffusion controlled drug delivery. J. Control. Release 161, 351-362. doi:10.1016/J.JCONREL.2011.10.006

Terrin, G., Canani, R.B., Chiara, M.D., Pietravalle, A., 2015. Zinc in early life: a key element in the fetus and preterm neonate. Nutrients 7, 10427-10446.

Vir, S.C., 2011. Public Health and Nutrition in Developing Countries. CRC Press.

World Health Organization, 2015. WHO Model List of Essential Medicines for Children 5th list.

World Health Organization, 2007. Promoting safety of medicines for children.

World Health Organization, 2017. Infant and young child feeding, Fact sheet N. 342. http://who.int/mediacentre/factsheets/fs342/en/. (accessed 02.01.16), n.d.

Wulf, K., Wilhelm, A., Spielmann, M., Wirth, S., 2013. Frequency of symptomatic zinc deficiency in very low birth weight infants. Klin. Paediatr. 225, 13-17.

Yoo, H.S., Kim, T.G., Park, T.G., 2009. Surface-functionalized electrospun nanofibers for tissue engineering and drug delivery. Adv. Drug Deliv. Rev. 61, 1033-1042. 